\title{
Machine Learning Agents in the Cloud to Support Smart Business Process Management
}

\author{
Samia Gamoura ${ }^{1}$, Laurent Buzon $^{2}$, Ridha Derrouiche ${ }^{3}$ \\ ${ }^{1}$ Agenor-I, 1, Place Colonel Fabien, 69700 Givors, France \\ samia.gamoura@gmail.com \\ ${ }^{2}$ ESCE - International Business School, 6 cours Albert Thomas BP 8242 - 69355 Lyon \\ Cedex 08, France \\ laurent.buzon@esce.fr \\ ${ }^{3}$ EVS-UMR5600, 158 cours Fauriel, 42023 St-Etienne Cedex 2, France \\ ridha_derrouiche@esc-saint-etienne.fr
}

\begin{abstract}
In Virtual Enterprise, Business Processes Management is regarded as one of the most concerns of managers and academic researchers. Managing flows complexity and actors requirements in terms of high quality in less time, make this management more and more complex and push specialists to explore new promising ways. Like these researchers, we present in this paper, a modelling and simulating software toolkit called BP-EMC ${ }^{2}$ based on a generic framework baptized H-BPM. We propose a solution using machine learning agents operating in an AGR (Agent-Group-Role) organization within the Cloud. Furthermore, this paper includes a real case study of Adecco ${ }^{\circledR}$ business process deployed into its Cloud solution.
\end{abstract}

Keywords: Business Process, Multi Agent System, Virtual Enterprise, modelling, simulation, Cloud Computing, Machine Learning.

\section{Introduction}

Since the beginning of the 90 's, inter-enterprises collaboration stills growing and generates new organizations patterns. Today, competition is no more limited in the internal organization but is extended in the whole enterprises network. These new kind of relationships created is called Virtual Enterprises (VE) focused on sharing Business Processes (BP). Here we situate our purpose to address an important issue in Business Processes Management (BPM): 'Collaboration'. The main topic considered in this paper is according to the collaboration strategies and how these strategies could be deployed overall BPM within Virtual Enterprise Network (VEN).

One from the huge number of methods and techniques used as a support for collaboration in VEN is Multi Agents Systems (MAS). As in many other business areas, such as medical, image processing, astronomy, etc. this paradigm of Distributed Artificial Intelligence (DAI) provided an efficient way to propose solutions and simulation of complex issues. Furthermore, we explain a new advanced kind of agents called Machine Learning Agents (MLA), able to use Machine Learning (ML) 
algorithms efficiently within the Cloud environment in order to extract and predict the best way of providing collaboration to companies.

\section{Business Processes in Virtual Enterprises}

VEs can achieve their business objectives only through effective collaboration between the autonomous enterprises that comprise them. A key requirement for theses VEs is called 'Collaborative BP' that explicitly captures and manages the functional and contractual relationships between VE's partners.

\subsection{Virtual Enterprises Networks}

As a formal definition, VEN is a temporary alliance of enterprises that aim to share resources and skills in order to respond better and faster to emerging opportunities in the market, based on a technical infrastructure and information technologies [1].

In Fig. 1. we illustrate the VEN concept with a network of companies created to design and manufacture a new type of stool [2].

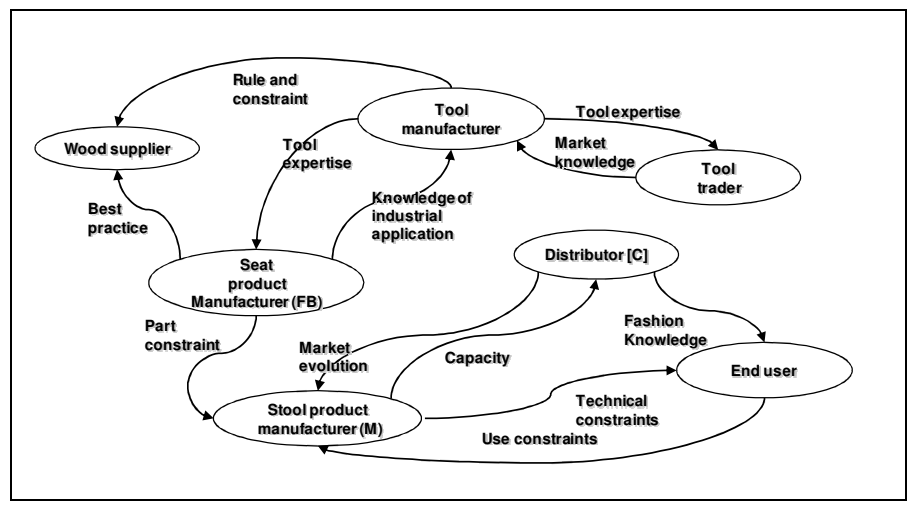

Fig. 1. An example of VEN of a stool Supply chain

The next section deals with the essential elements of BPM to identify concepts and approaches that aim to design an efficient tool to help VEN partners to manage and improve their cooperation.

\subsection{Business Processes Management Methodologies}

As mentioned by the Object Management Group (OMG) in [3], the primary goal of BPM modelling is to provide a notation that is readily understandable by all business users, from the draft of the initial business analyst, to the technical developers responsible for implementing the technology that will perform those processes, and finally, to the business people who will manage and monitor those processes. The 
state-of-the-art shows three main modelling methodologies: One-Thing Approach (OTA) [4], View-Based Approach (VBA) [5] and Holistic Process Methodology (HPM).

The main idea behind the HPM approach is that domain specific process models are required both to capture the contents of a process based application and to present a process model in a user-friendly way. The assumption is that this application is composed of single activities that have to be executed in a certain order [6].

According to our framework, we choose to use this last methodology for several reasons: agility, wide usage and user-friendly feature to insure business people requirements.

\section{Multi Agent Systems and Machine Learning}

Systems based on a set of software agents commonly named MAS have been used widely following an exponential trend during recent ten years, and this is mainly due to their potential in understanding various group behaviors such as swarms and flocking. In industry and business applications, in domains as several as manufacturing [7], war gaming [8], satellite clusters [9] and so on. Furthermore, a number of development environments and frameworks are actually available, i.e. $\mathrm{SWARM}^{\circledR}$ [10], JADE ${ }^{\circledR}$ [11], JACK ${ }^{\circledR}$ [12], etc. appeared. Computer science architects and program designers believe that this paradigm called Agent Oriented Programming (AOP) is the next step forward from Object Oriented Programming (OOP) [13].

In the following section, we begin by positioning MAS in terms of its business uptake. We know that there is a lot of research works, ideas, and projects, but the uptake of the technology is not as rapid or as pervasive as its advocates anticipated [13]. In the configuration MAS-BP, one of the bright works is that one of Fleischermann et al. in [14]. According to authors, the emerging paradigm of the subject oriented BPM by MAS is to augment MAS models with a process-centric layer that preserves autonomy and concurrent interactions. Another remarkable work was published by Bonfante et $a l$. in [15] inspired by the same principle of the MAS layer above the process layer. The proposed architecture is composed of a layer between BP and Web canals.

\subsection{Agents and Predictive Machine Learning Algorithms}

Research works, publications, books, projects, applications in the area of Machine Learning Agents (MLA) are numerous, especially in recent works concerned by Big Data phenomenon: [16], [17], etc. ML techniques can be classified according on several criteria to several classes. These techniques are to be coupled to learning classes according to the data nature where the learning type could be supervised or unsupervised. 
The most widely used technique is the last one; Q-Learning; also called Reinforcement Learning (RL), easy to implement and convergence is guaranteed. This is one of the reasons that push us choosing it to predict our agents' actions.

\section{Cloud Computing in Business Process Management Context}

Nowadays, Cloud computing is the fundamental change happening in the field of IT. The special report published by the National Institute of Standards and Technology (NIST) in U.S. [18] provides a clear understanding of Cloud computing. It provides a simple and unambiguous taxonomy of three service models available to Cloud consumers: Cloud Softwares As A Service (SaaS), Cloud Platform As A Service (PaaS) and Cloud Infrastructure As A Service (IaaS).

This paper presents an organized snapshot of the challenges faced by scientists and professionals in designing and deploying BPM within VE in the Cloud. Our background study encompasses both classes of systems: for supporting update dynamic BPM and for ad-hoc analytics and decision support using intelligent tools within SaaS Cloud platforms.

Since 2010, big numeric companies propose industrial software products for BPM in the Cloud, such Oracle Fusion Middleware $11 \mathrm{~g}$ by Oracle ${ }^{\circledR}$ [19]. A 'Cloudified' BPM allows what is called 'Unified Collaboration' or 'Social BPM' which becomes increasingly more and more important, especially with the IT virtualization phenomenon. Distributed nature of Cloud reinforces the need for process visibility and collaboration, especially as systems and people are distributed. Also, BPM should leverage optimized structure and management of 'Cloudification'.

All these reasons have guided our decision to locate our platform on the Cloud, with services offered in SaaS with appropriate rights management. This particular part of rights management and security will be presented in further publications topics.

\section{Framework Proposal BP-EMC ${ }^{2}$}

In this work, we look at the issue of BPM in VE of nowadays, with new challenge of 'Cloudification'. As we already introduced, we have chosen to automate the dynamic monitoring of changes in processes by AGR machine learning agents (Fig. 2.). 


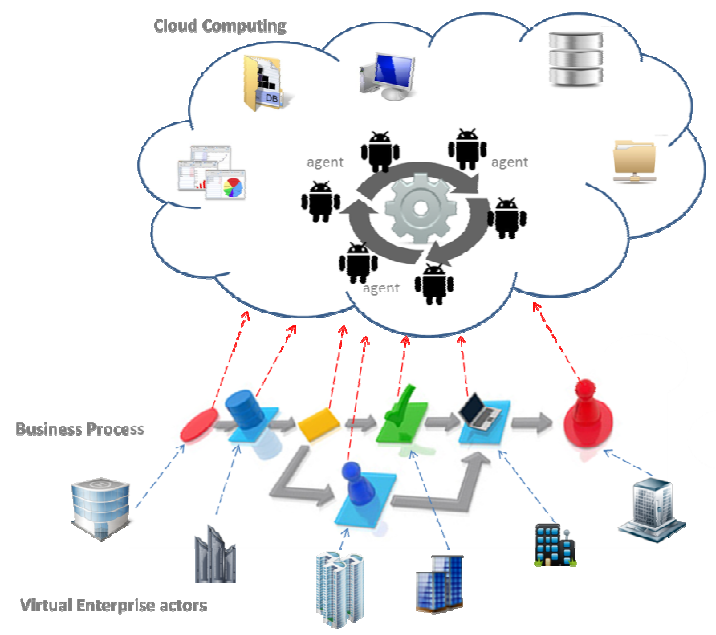

Fig.2. Machine Learning Agents in the Cloud for BP-EMC 2

For experimental needs and validation, we worked on an industrial case study of Adecco ${ }^{\circledR}$ recruitment process shared with its partners; HP ${ }^{\circledR}$ and Renault ${ }^{\circledR}$ in a VE context (we call this VE Adecco ${ }^{\circledR} \&$ Co.). In the following sections, we will describe step by step how we design and build our solution by using this case study, without exposing deep details.

\subsection{Building Smart BPM Model in the Cloud}

We regard a process as a collection of activities that consume some inputs in order to produce some outputs based on HPM (as discussed previously in section 2.2) in the process life management as detailed in the following steps:

Step 1. Managing Process Life Cycle (PMLC) by Holistic Approach (H-BPM): The BPM life cycle is constituted by a set of activities and the model has to define a clear guideline how a process must be performed. To design our model named HBPM, our concern was to notice all key concepts in the life cycle model offered in HPM methodology. Thus, we consider some of them:

- Item: three types of items; Actor (a reactive or proactive entity which can act and initiate an activity of launch events, such as human task, automatized task, application module, etc.), Object (a passive entity which is handled by actors, such messages, documents, products, etc.) and Event (all events defined in BMPN such as 'timer', 'start and 'end' events).

- Configuration: a set of items with related parameters define a configuration. It is the set of all the elements contained in the BMPN relative to a time reference.

- Time guide-based: The life cycle is marked by a time stamp. During this cycle, multiple configurations may exist following time. 
- Version: each item in the configuration is mentioned by a version, and the configuration itself is versioned.

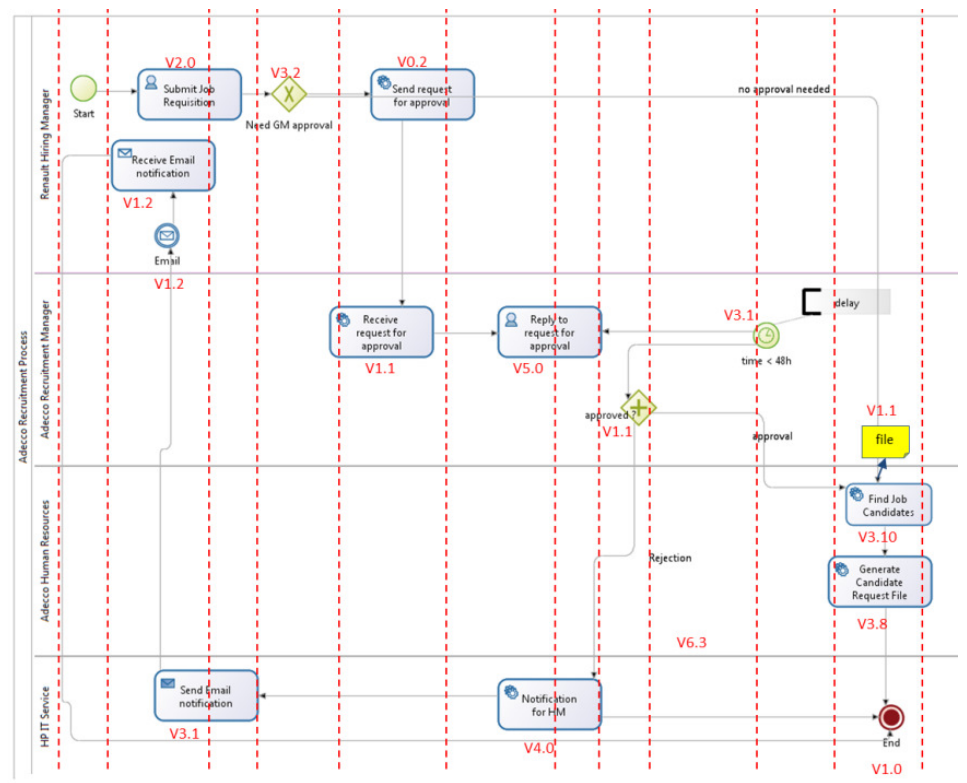

Fig. 3. Recruitment BPM in Adecco ${ }^{\circledR}$ \& Co. modeled by H-BPM (by IBM-Bonitasoft ${ }^{\circledR}$ 6.5.1)

Step 2. Introducing Machine Learning algorithm in H-BPM agents' behavior: As discussed previously, we introduce a Machine Learning algorithm to give ability to our agents to be adaptive and predictive to augur better actions to help human and system. We implement ML based on Q-Learning algorithm where each agent has a history as a sequence of state-action-rewards.

Step 3. Modelling H-BPM components: Our built model is based on autonomous agents, with whom we aim to understand and study the behavior actors withinBPM. In our proposed platform, agents are necessary to initiate and drive the execution of processes. Based on key concepts described previously, we design our system components based on three main entities: Actors, Events and Objects.

Pools in H-BPM should be designed as 'Roles' entities. And agents indicates 'Actors', Objects are designed by 'Objects' and finally events are defined as 'Events' for agents' input/output flows. Actions performed by actors in H-BPM are implemented in 'Roles' (Fig. 5.). 


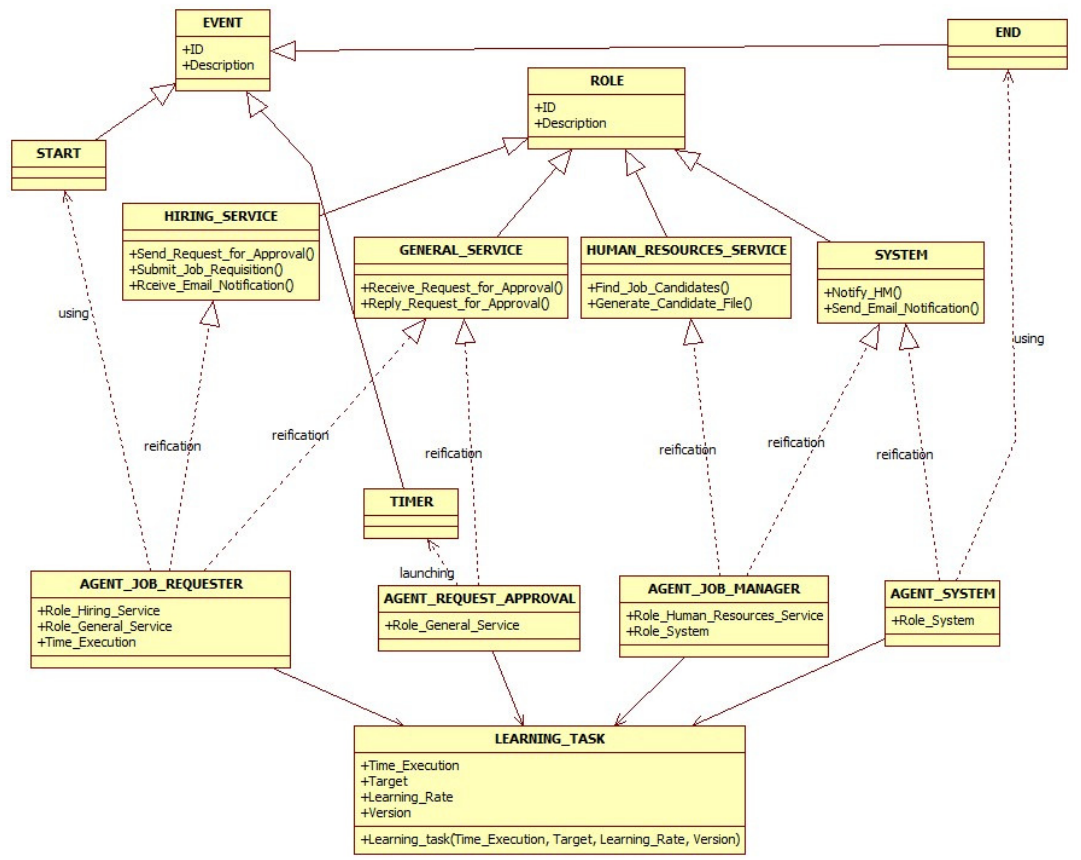

Fig.5. Learning with AGR in H-BPM (by StartUML 5.0.3 ${ }^{\circledR}$ )

Based on this presented model H-BPM, we baptize a software toolkit BP-EMC ${ }^{2}$ as 'Business Process - Evolutive Management by Collaborative agents in the Cloud'. The remaining part in the next section is devoted to the description of this toolkit.

\section{Experimental Validation of BP-EMC ${ }^{2}$}

The software platform was designed and conducted in JAVA ${ }^{\circledR}$. Following the study of both platforms SWARM ${ }^{\circledR}$ and $\mathrm{JADE}^{\circledR}$, this last was chosen because of the following advantages: it is a platform FIPA compliant (meaning feature ensures interoperability with protocols and standards); it manages the task achieving parallelism; it allows reusability and all imported libraries are free software.

\subsection{BP-EMC ${ }^{2}$ Features: Case Study Adecco ${ }^{\circledR} \&$ Co. Recruitment BPM}

Our toolkit named BP-EMC ${ }^{2}$ is a generic model of BPM with agents as we conceive it. The framework embeds two modules: 'Modelling module' where we can configure the system and the 'Simulation module' which is a Graphical User Interface (GUI) that uses the parameters defined in the model described in the previous section for 
illustration, analysis and numerical and graphical monitoring of experimental scenarios.

During our simulations campaigns, our main objective was to provide proofs that agents in BP-EMC ${ }^{2}$ have the ability to improve overall BPM in terms of time execution and targets. Using predictive agents in the Cloud should provide a high target level with short time execution. Thus, we considered described metrics: Time Execution (TE) and TarGet (TG) according to time-line represented by Versions (V).

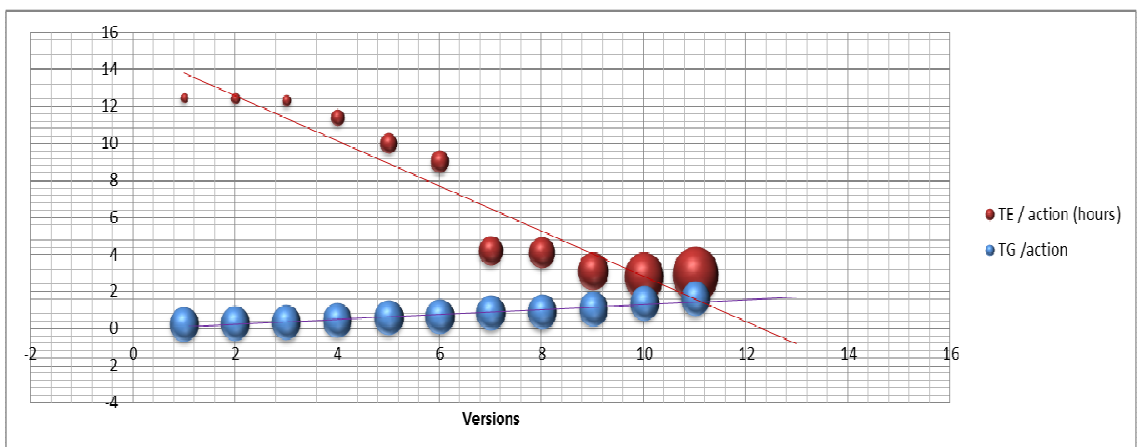

Fig.8. Training for Agent_Job_Requester in BP-EMC ${ }^{2}$ (50 iterations) (with $\gamma=0.8$, $Q^{0}[0], 11$ versions, 17 days)

In order to compare our proposition with a solution without agents, we refer to SAS $^{\circledR}$ (the Business Intelligence (BI) software) extracted Key Performance Indicators (KPI) data in figures below. Used KPI are: 'AD KPI' as 'Action Duration KPI' (cf. Fig. 9.) and ' $A Q K P I$ ' as 'Action Quality KPI' (cf. Fig. 10.).

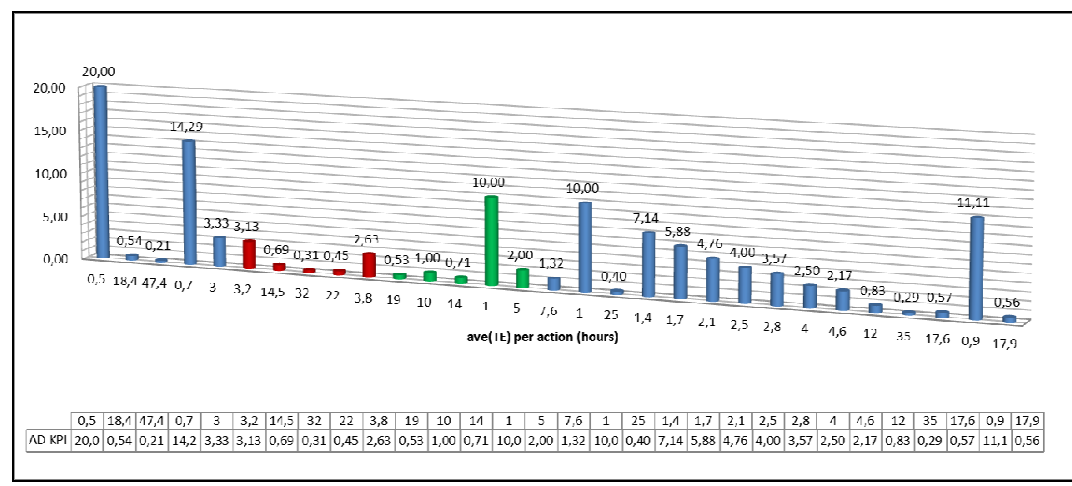

Fig. 9. Extraction of $A D K P I$ for a month (30 days) (by SAS ${ }^{\circledR}$ Software) 


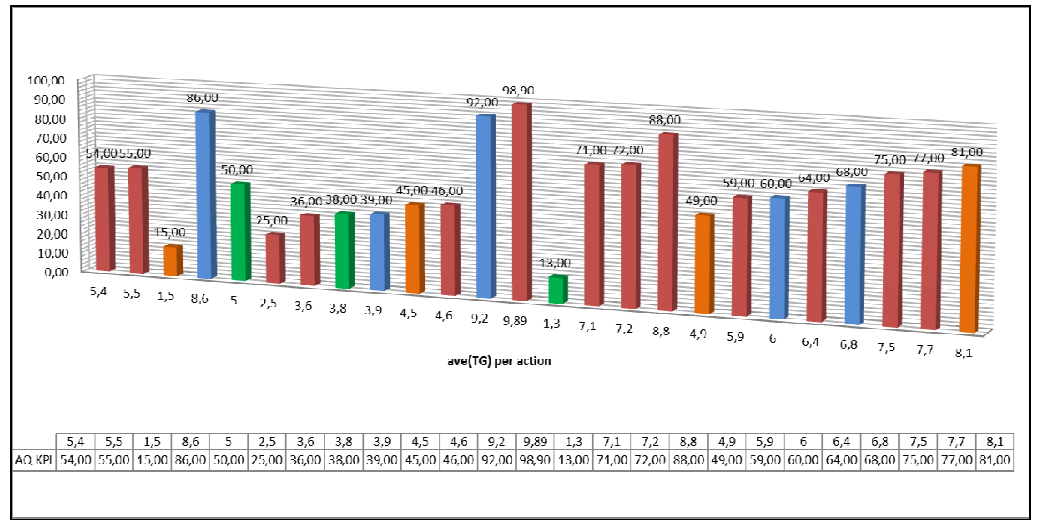

Fig. 10. Extraction of $A Q K P I$ for a month (30 days) (by SAS ${ }^{\circledR}$ Software)

\subsection{Discussion}

As we see on the both trend curves in figure Fig. 8, both indicators $T E$ and $T G$ tend to stabilize and to optimize values. When, after several iterations we get close values, we speak of stability. At the $11^{\text {th }}$ iteration, the average execution time of the action Agent_Job_Requester is 2.9 hours with a TG/action indicator of $0.08 / 10 \sim 80 \%$, and this is satisfactory and maintained for next versions. Then, if we compare this (Fig. 8) with extractions in figures Fig. 9 and Fig. 10, we can notice that the average time per action moy(TE)/action may be slightly more interesting ( 0.5 to 1 episode) but with a mediocre quality indicator $T G$ of $5.5 / 10 \sim 55 \%$ in longer global time execution (30 days vs 17 days). Also, we have not insurance that this will be maintained as there is no system for experience storage and management (no memory). As we see, these values vary and may worsen from an episode to another.

These results are admittedly preliminary but very promising: Learning agents helped refine the convergence to a more optimal BPM model. This convergence is reached after a number of versions obtaining a short execution time with a high quality indicator. These results may be similar to a conventional BPM (without the use of Machine learning agents Results), but can take more time longer and without any insurance of stability.

\section{Conclusion}

This paper attempts to address the issue of collaboration in the area of Virtual Enterprises which concerns Business Process Management under Cloud Computing requirements. As technology evolves, flexibility of BPM adapts to new requirements. Processes can be managed at anytime from anywhere and optimization allows to adjust processes to Cloud SaaS context. Our toolkit called BP-EMC ${ }^{2}$ (Business 
Process - Evolutive Management by Collaborative agents in the Cloud), tries to provide a complete platform for BPM partners to face new IT challenges. We believe that, our work may be an efficient tool to reduce complexity and provides the flexibility required for any BPM in Virtual Enterprise.

\section{References}

1. Roşu, S.M., Drāgoi, G.:Virtual enterprise network general architecture. In: 8th IEEE International Conference on Communications (COMM), pp: 313 -- 316 . IEEE Press, New York (2010).

2. Buzon, L., Bouras, A., Ouzrout, Y.: Knowledge exchange in a supply chain context, in: IFIP 18th World Computer Congress, TC5 / WG5.5 : PRO-VE'04, 5th Conference on Virtual Enterprises, pp:145-152 Ed. Kluwers Academics Publishers, Dordrecht (2004).

3. Business Process Model and Notation (BPMN), Version 2.0. (2011), http://www.bpmn.org/

4. Vogel, O., Arnold, I., Chughtai, A., Kehrer, T.: Software Architecture: A Comprehensive Framework and Guide for Practitioners. Springer, Heidelberg (2011).

5. Hentrich, C., Zdun,: Patterns for Process-Oriented Integration in Service Oriented Architectures, In: $11^{\text {th }}$ European Conference on Pattern Languages of Programs, (2006).

6. Dori, D.: Object-Process Methodology: A Holistic Systems Paradigm. Springer, Heidelberg (2002).

7. Liu, Q., Gao, L., Lou, P.: Resource management based on multi-agent technology for cloud manufacturing. In: IEEE 2011 International Conference on Electronics, Communications and Control, pp. 2821-2824, IEEE press, New York (2011).

8. Tan, X., Lai, S., Wang, W., Zhang, M.: Framework of wargame CGF system based on multi-agent. In: IEEE International Conference on Systems, Man, and Cybernetics. pp. 3141-3146. IEEE press; New York (2012).

9. Schettera, T., Campbella, M., Surkab, D. : Multiple agent-based autonomy for satellite constellations. Journal of Artificial Intelligence, 145, 147--180 (2003).

10. SWARM ${ }^{\circledR}$ Project under GNU Lisence. http://www.swarm.org

11. JADE ${ }^{\circledR}$ Framework under LGPL Lisence. http://jade.tilab.com

12. JACK ${ }^{\circledR}$ Software under AOS Group Lisence. http://aosgrp.com/products/jack

13. Jarvis, D., Jarvis, J., Rönnquist, R., Lakhmi C.: Multiagent Systems and Applications. Springer, Heidelberg (2013).

14. Fleischmann, A., Metasonic AG, Pfaffenhofen, G., Kannengiesser, U., Schmidt, W., Stary, C.: Subject-Oriented Modelling and Execution of Multi-agent Business Processes. IEEE International Joint Conferences on Web Intelligence (WI) and Intelligent Agent Technologies (IAT). vol.2, pp. 138-145. IEEE press; New York (2013).

15. Bonfante, M. C.,Paz, J. P., Castillo, A.: Multi-Agent System for Integration Process Business and Ontologies for the Government Online Strategy. International Journal of Applied Science and Technology,. 7(4), 19--25 (2014).

16. Rand, W.: Machine learning meets agent-based modelling: when not to go to a bar. Conference on Social Agents: Results and Prospects (2006)

17. Kotsiantis, S.B.: Supervised MachineLearning : A Review of Classification Techniques. Journal of Emerging Artificial Intelligence Application in Computer Engineering. IOS Press, Amsterdam (2007).

18. Liu, F., Tong, J., Mao, J., Bohn, R., Messina, J., Badger, L., Leaf D.: NIST Cloud Computing Reference Architecture. NIST Special Publication (2011).

19. Connaughton, M.: Business Process Management and Cloud Computing. Oracle Middleware 11g Forum (2011). 